\title{
LA TOMOGRAFÍA DE COHERENCIA ÓPTICA, UN PUNTO DE INFLEXIÓN EN NEURO-OFTALMOLOGÍA
}

\section{OPTICAL COHERENCE TOMOGRAPHY, AN INFLECTION POINT IN NEURO-OPHTHALMOLOGY}

\author{
REBOLLEDA G $^{1}$, MUÑOZ-NEGRETE FJ ${ }^{1}$
}

\begin{abstract}
Quien iba a decirle a Santiago Ramón y Cajal que apenas un siglo después de su «doctrina de la neurona», que le hizo merecedor del Nóbel de Medicina, pudiéramos cuantificar «in vivo» la capa de fibras nerviosas de la retina (CFNR) de una forma no invasiva, sencilla, rápida, objetiva, reproducible $\mathrm{y}$ altamente resolutiva.
\end{abstract}

La tomografía de coherencia óptica (OCT) se ha ido convirtiendo en una herramienta cotidiana y muy valiosa, tanto para el diagnóstico como para la monitorización y comprensión de diversas entidades, dentro del marco de la neuro-oftalmología. Quizá una de las patologías donde más nos ha aportado es en la neuritis óptica. La OCT no ha venido sino a confirmar que un proceso desmielinizante asocia a menudo daño neuronal o muerte axonal.

Son varios los estudios que han demostrado un adelgazamiento significativo de la CFNR peripapilar tras un brote de neuritis óptica tanto en formas monosintomáticas o aisladas, como en el contexto de una esclerosis multiple (1-5). La CFNR no es otra cosa que el conjunto de axones no mielinizados de las células ganglionares, y esto justifica que cuanto mayor sea el adelgazamiento de la CFNR, en definitiva cuanta mayor sea la muerte neuronal, mayor también será el déficit visual; y por lo tanto que exista una correlación significativa entre el grado de adelgazamiento y la pérdida de sensibilidad al contraste, así como con la reducción de la amplitud de los potenciales evocados visuales. Respecto a la perimetría automática, no son raras las discrepancias, de modo que adelgazamientos de la CFNR no siempre tienen su correspondiente defecto funcional; pudiendo en estos casos inferirse una mayor sensibilidad diagnóstica en el caso de la OCT.
La pérdida de fibras conlleva un incremento de la excavación, incremento que es tanto más acentuado cuanto mayor es la pérdida axonal, y por lo tanto más común con peores agudezas visuales. El hallazgo de una excavación patológica clínicamente relevante se puede objetivar hasta en una quinta parte de los casos. En estos casos, tanto los antecedentes clínicos como la palidez del anillo neurorretiniano, plantean pocas dudas en el diagnóstico diferencial con otras neuropatías excavadas.

La cuantificación del edema papilar ha demostrado que no existen diferencias en cuanto al grado de déficit visual entre formas retrobulbares y formas anteriores o papilitis; y dentro de estas últimas tampoco hay diferencia en el grado de recuperación en función de la intensidad del edema en fase aguda; de modo que el grado de edema no tiene valor predictivo respecto a la recuperación visual (3).

Pero los hallazgos encontrados en OCT no se limitan al marco oftalmológico, y así esta muerte axonal se produce también en el contexto de una esclerosis múltiple, incluso en pacientes sin antecedentes de neuritis óptica; si bien el adelgazamiento de la CFNR en estos casos es menos acentuado. Se ha visto además que dicho adelgazamiento está íntimamente relacionado con la duración de la enfermedad, con el grado de deterioro o discapacidad neurológica, con el número de recaídas, y con la atrofia objetivada mediante resonancia magnética $(1,5,6)$.

Teniendo en cuenta todos estos datos, parece obligado que cualquier ensayo o estudio dirigido a evaluar el papel de los diversos agentes terapéuticos existentes o emergentes dirigidos a neuroproteger tanto en neuritis óptica como en esclerosis múltiple,

\footnotetext{
1 Doctor en Medicina. Hospital Ramón y Cajal. Universidad de Alcalá. Madrid. España.

E-mail: franciscojmunoz@telefonica.net
} 
incluyan entre sus evaluaciones los resultados de la OCT antes y después del tratamiento $(7,8)$.

La OCT no solo nos permite cuantificar o medir la CFNR, sino también analizar las características morfológicas del disco óptico. En este sentido hemos podido comprobar que el «disco de riesgo» típicamente asociado a la neuropatía óptica isquémica no arterítica (NOINA), no se corresponde con una papila pequeña, como clásicamente ha sido referido. Parece que el aspecto de disco amontonado y la ausencia de excavación se deben a una posición más anterior de la lámina cribosa. En este contexto clínico, de modo similar a como sucedía en la neuritis óptica, la pérdida axonal se traduce en un aumento de excavación, que por ser menos llamativa pasa clínicamente desapercibida $(9,10)$.

Otra entidad en la que la OCT es muy útil es en el seguimiento del papiledema, ya que nos permite el análisis cuantitativo y seriado del edema papilar, amén de la valoración de las posibles secuelas. Pese a esto, no es útil para diferenciar el edema papilar real del «pseudoedema» (11).

No es nuestra intención enumerar todas aquellas neuropatías en las que la OCT puede ser de alguna utilidad, pero sí recordar que nos brinda una oportunidad única al permitir una biopsia «in vivo»y altamente resolutiva, menos dependiente de la colaboración del paciente que la perimetría.

Todo esto no ha hecho más que empezar; la resolución de 10 micras de los tomógrafos habituales ya está siendo reemplazada por resoluciones de 2-3 micras; y antes de que nos demos cuenta estaremos explorando a nuestros pacientes con resoluciones inimaginables en la actualidad.

\section{BIBLIOGRAFÍA}

1. Kallenbach K, Fredericksen J. Optical coherence tomography in optic neuritis and multiple sclerosis: a review. Eur J Neurol 2007; 14: 841-849.

2. Costello F, Coupland S, Hodge W, et al. Quantifying axonal loss after optic neuritis with optical coherence tomography. Ann Neurol 2006; 59: 963-969.

3. Noval S, Contreras I, Rebolleda G, Munoz-Negrete FJ. Optical coherence tomography versus automated perimetry for follow-up of optic neuritis. Acta Ophthalmol Scand 2006; 84:790-794.

4. Fisher JB; Jacobs DA, Markowitz CE, et al. Relation of visual function to retinal nerve fiber layer thickness in multiple sclerosis. Ophthalmology 2006; 113: 324-332.

5. Sepulcre J, Murie-Fernandez M, Salinas-Alaman A, Garcia-Layana A, Bejarano B, Villoslada P. Diagnostic accuracy of retinal abnormalities in predicting disease activity in MS. Neurology 2007; 68: 1488-1494.

6. Trip SA, Schlottmann PG, Jones SJ et al. Optic nerve atrophy and retinal nerve fibre layer thinning following optic neuritis: evidence that axonal loss is a substrate of MRI-detected atrophy. Neuroimage 2006; 15; 31: 286-293.

7. Rebolleda G, Muñoz-Negrete FJ, Noval S, Contreras I, Gilbert ME, Sergott RC. New ways to look at an old problem. Surv Ophthalmol 2006; 51: 169-173.

8. Sergott RC, Frohman E, Glanzman R, Al-Sabbagh A: on behalf of the OCT in MS Expert Panel. The role of optical coherence tomography in multiple sclerosis: Expert panel consensus. J Neurol Sci. 2007 Jul 26; [Epub ahead of print].

9. Contreras I, Rebolleda G, Noval S, Muñoz-Negrete FJ. Optic disc evaluation by optical coherence tomography in nonarteritic anterior ischemic optic neuropathy. Invest Ophthalmol Vis Sci 2007; 48: 4087-4092.

10. Contreras I, Noval S, Rebolleda G, Muñoz-Negrete FJ. Follow-up of Nonarteritic Anterior Ischemic Optic Neuropathy with Optical Coherence Tomography. Ophthalmology 2007 Aug 24; [Epub ahead of print].

11. Karam EZ, Hedges TR Optical coherence tomography of the retinal nerve fibre layer in mild papilloedema and pseudopapilloedema. Br J Ophthalmol 2005; 89:294-298. 